\title{
The heated humidification system for respirator based on PID
}

\author{
Chen Xue-fang \\ Computer school, Dongguan university of Technology, Dongguan, China \\ e-mail: chenxf@dgut.edu.cn
}

\begin{abstract}
To provide patients with a comfortable and healthy breathing environment, a heated humidification system for respirator is designed by PID (Proportional, Integral and Differential) algorithm and silicon controlled rectifier (SCR). Zero-crossing detection method is also introduced, and its circuit is also designed to realize PID algorithm. The testing experiment was established, temperature stability of the system was tested. Result shows that the system is responsive for stable temperature control, and easy to transplant.
\end{abstract}

Key words: PID; SCR; heated humidification; respirator; Zero-crossing detection

\section{Introduction}

To provide comfort breathing environment to the patients and avoid dry cold air damage to the respiratory tract and lungs, the inhaled gas should be wetted and heated in the respirator treatment. Studies have shown that: the respirator can effectively avoid the allergic uncomfortable conditions such as mouth, nose and throat being dried, sneezing, nasal congestion and runny nose in the process of use when increase a humidifier. Modern commonly used two methods: one is the atomization humidification; one is the steam humidification. The former is by using the principle of jet, which make water droplets collision into tiny particles suspended in the suction gas; its disadvantage is that atomized particles is not fine enough, breathing gas heating not easily, wetting degree is difficult to control, jet nozzle clogs easily and moist effect is not stable. The latter is to heat water into steam, sneak into the inhalation gas to achieve the dual purpose of humidification and heating, but the heating temperature is affected by many factors such as ambient temperature, the length of the airway and the disorders of temperature control system, causing higher or lower temperature, resulting in adverse effects on the patients and even burn the respiratory tract. If there is a problem of the humidifier, not only cannot play the desired effect, but may damage the patient's respiratory(Such as the humidifier's temperature out of control can make patient's respiratory burn, etc). ${ }^{[1-6]}$ Therefore, humidifier plays an important role in the patient's respiratory therapy.

PID controller is used widely in kinds of industry circumstance for its simple structure, easy implementation and strong robustness. ${ }^{[7-9]}$ This paper presents a heated humidification system for respirator based on PID, which has many characteristics such as temperature can be adjusted, responsive and stable temperature control.

\section{Design of circuit program}

On the basis of the traditional heated humidification system for respirator, join the PID algorithm for temperature control. The system includes: MCU control chip, the heating plate, the zero-crossing detection circuit, the control of PID parameters and the bidirectional triode thyristor. Fig. 1 is a hardware diagram frame of the system, which is composed of the control module, the drive module, the load module and the display module.

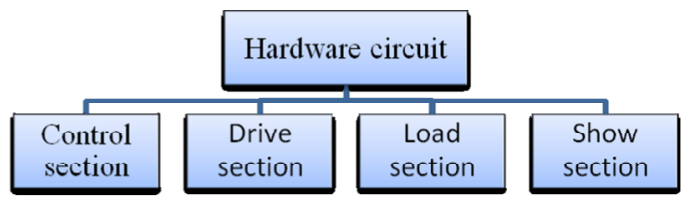

Figure1. The hardware diagram frame of the heated humidification system

The control module: In order to facilitate flexible design, choosing programmable devices which can write many times, such as microcontroller or ARM chips. In order to reduce cost, the main chip which controls the respirator can be adopted. This system uses the respirator control chip STM32F103ZE directly.

The drive module: This system adopts the optical coupler (OC) and bidirectional triode thyristor to drive, 
including: zero-crossing detection circuit and SCR circuit. This section is fundamental to achieve PID control. OC transmit electrical signals by light as the medium, the input and output electrical isolation completely, strong anti-jamming capability, stable work, high transmission efficiency. The SCR enables non-contact control AC, a small current to control a large current, and it is fast, long life and high reliability.

The load module: The system uses the operating voltage $220 \mathrm{~V}$, rated power of $150 \mathrm{~W}$ heating plate.

The show module: The system uses TFT display shows the set temperature and the actual temperature.

The feature of SCR is that even the trigger signal is removed after conduction, it will still keep conduction, when the load current is zero (AC voltage zero point), it will automatically turn off. This system is to be controlled heating plate, its working voltage: $220 \mathrm{~V} / 50 \mathrm{~Hz}$. Take the zero-crossing signal (synchronization) of sinusoidal alternating through $\mathrm{OC}$ and the signal is sent to the external interrupt control chip MCU. The moment MCU control chip received synchronization signal, determine whether to generate a trigger signal based on real values by the PID algorithm. When the MCU control chip generates a trigger signal, namely make the SCR conduction, current flowing through the heating plate through SCR, so that make the heating plate work. Determine how much trigger signal is needed to generate according to the PID algorithm in a period which is $\mathrm{N}$ sync signal. The more trigger signal the MCU control chip generated, the longer the heating plate is heated, the higher the temperature is, on the contrary, the shorter the heating plate is heated, the lower the temperature is.

\section{Implementation of PID regulation}

\subsection{PID algorithm introduction}

The traditional PID control is according to the given value $\mathrm{r}(t)$ and the actual output value $y(t)$ to get control deviation $e(t)$, namely, $e(t)=r(t)-y(t)$, to operate the deviation $e(t)$ with proportion, integral and differential, and then combined the results of operation, get the output $u(t)$ from the PID controller, the PID control system block diagram shown in Fig. $2 .^{[7]}$

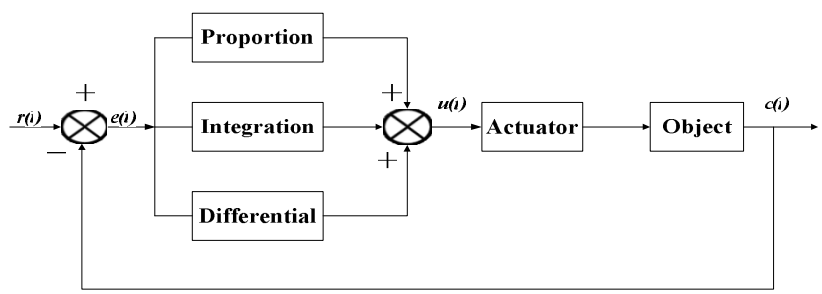

Figure2.The PID control system block diagram

In the digital control system can get the discrete PID expression is:

$$
u(k)=k_{p}\left\{e(k)+\frac{T}{T_{i}} \sum_{j=0}^{k} e(j)+\frac{T_{d}}{T}[e(k)-e(k-1)]\right\}
$$

In the equation (1), $k_{p}$ is a scale factor, $T_{i}$ is the integration time constant, $T_{d}$ is the derivative time constant, $\mathrm{T}$ is the sampling period.

Equation (1) can be simplified as:

$u(k)=k_{p} e(k)+k_{i} \sum_{j=0}^{k} e(j) T+k_{d} \frac{e(k)-e(k-1)}{T}$

In the equation (2), $k_{p}$ is a scale factor; $k_{i}$ is an integral coefficient, $k_{i}=k_{p} / T_{i} ; k_{d}$ is a differential coefficient, $k_{d}=k_{p} * T_{d} ; \mathrm{T}$ is the sampling period.

Various parameters of PID controller plays a different role on dynamic and steady-state performance of the system, the merits of these three parameters' values will directly affect the controlling quality of the PID control system. The role of the various correction parts of the PID controller as follows:

Proportional part: Once the deviation signal $e(t)$ of reaction control system which is proportional generated, the controller generates a control action immediately to reduce the deviation. (1) Impact on the dynamic characteristics. Proportional control parameters $k_{p}$ increased, so that the system is more sensitive, faster. The number of oscillations increases and the adjustment time become longer when $k_{p}$ is bigger. When $k_{p}$ is 
too large, the system will tend to be unstable, and if $k_{p}$ is too small, the system will be slow. (2) Impact on the steady-state characteristics. Under the condition of the system is stable, if proportion coefficient $k_{p}$ increases, the steady-state error $e_{s s}$ will reduce and the control precision will improve. But increasing $k_{p}$ just reduce $e_{s s}$, could not essentially eliminate the steady-state error.

Integral part: mainly used to eliminate static error and improve the accuracy of the system. (1) Impact on the dynamic characteristics. Integral part will cause the stability of the system down. If $T_{i}$ is too small, the system will be unstable; else if $T_{i}$ is too big, the impact on system performance will be reduced. Only when $T_{i}$ is appropriate, there will appear ideal transition characteristics. (2) Impact on the steady-state characteristics. Integral part of the system can reduce the steady-state error, and also can improve the control accuracy of the system. However, when $T_{i}$ is too large, integral action will become very weak, steady-state error $e_{s s}$ will not be immediately reduced.

Differential part: shows the tendency of the deviation signal(rate of change), adjust the differential output of error in order to control timely when error mutations and can import an effective early correction signal to system before the error become too large, thus can accelerate the speed of system and reduce the adjusting time.

\subsection{Improvements on PID algorithm}

PID controller is widely used in industrial processes, but setting the parameters of PID controller is a vexing problem. This system proposes a new simple and effective method for parameter setting in view of the heated humidifier for respirator.

This system based on the discrete PID described above, make improvement from the following three aspects:

1. Add an external execution period;

2. The integral part execute conditionally in view of the heated humidifier for respirator, adding integral part until the temperature deviation signal $e(t)$ is greater than $0.5^{\circ} \mathrm{C}$.

3. Limit the executive scope of PID.

\section{System circuit and the concrete implementation}

\subsection{System circuit}

The drive section is the most important part of this system, which is fundamental to achieve PID control. This system adopts the OC and bidirectional triode thyristor to drive, including: zero-crossing detection circuit and SCR circuit.

Zero-crossing detection circuit is shown in Fig. 3. It is automatically detected when a sinusoidal AC zero crossing and generate sync signal. Fig. 4 is zero-crossing detection signal waveform, AC is $220 \mathrm{~V} / 50 \mathrm{~Hz}$, period is $20 \mathrm{~ms}$, after the bridge rectifier, negative phase change, period becomes $10 \mathrm{~ms}$, and namely, the period of zero-crossing detection signal is $10 \mathrm{~ms}$.

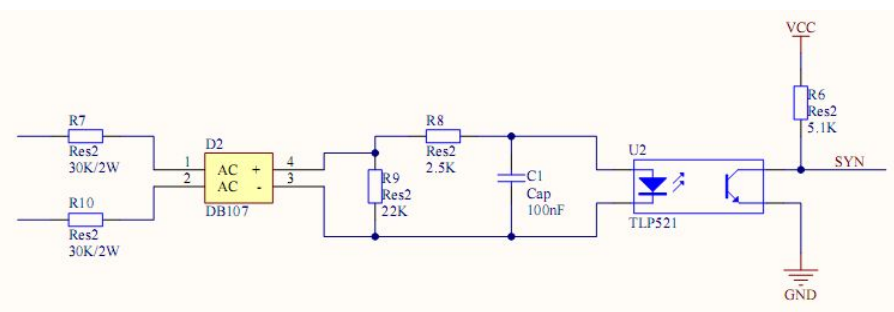

Figure3. Zero-crossing detection circuit

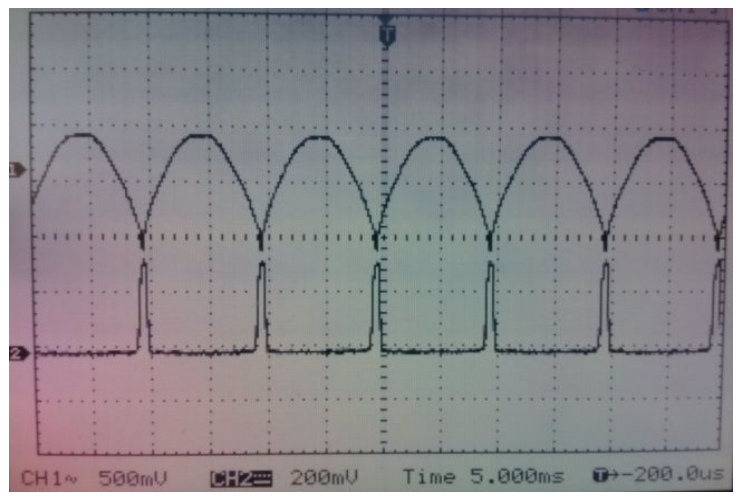

Figure4. Zero-crossing detection signal waveform

SCR circuit is shown in Fig. 5, this circuit is connected with the MCU and triggered by the trigger signal from MCU, thereby make the heating plate heat. Fig. 6 is SCR control signal waveform. 


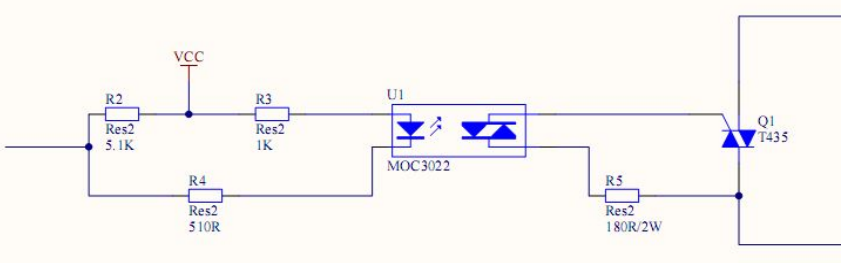

Figure5. SCR circuit

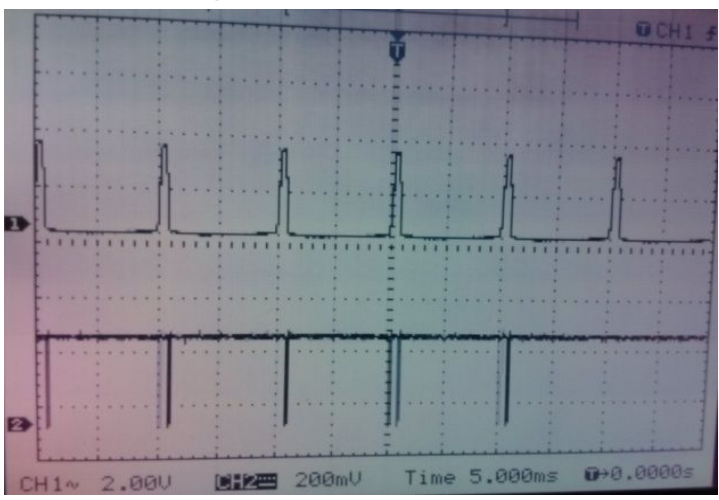

Figure6. SCR control signal waveform

\subsection{System implementation process}

The chip this system uses is STM32F103ZE, set the AD sampling time of 239.5 cycles, namely the PID sampling period is about 30us. Part 3.1 shows that the zero-crossing detection signal period is $10 \mathrm{~ms}$. PID sampling period is short, and the zero-crossing detection signal period is long, so PID operation updated about 333 times in a zero-crossing detection signal period. But the heating plate needs continuous heating, frequently updated PID can't let it work effectively. External interrupts recording the zero detection signal and $\mathrm{N}$ signal as a period, according to the characteristics of heating plate here selects 100 zero-crossing detection signal as an external execution period, PID algorithm is to convert temperature deviation value into corresponding SCR trigger signal, judge how many trigger signal need to give during $\mathrm{N}$ zero-crossing detection signal as a period. The external execution cycles of this system is 1s (100 zero-crossing detection signals). During this $1 \mathrm{~s}$, PID arithmetic updates about 33333 times and will feedback the result to the external interrupt. When the operation result is less than the last operation, SCR trigger and the heating plate work; on the contrary, SCR doesn't trigger and the heating plate doesn't work. Add external execution cycle can make heating plate work effectively, and at the same time execute PID update values sensitively. The size of external execution cycle is determined by the heating plate's features. If the heating plate heat up fast and have big power, the external execution cycle is small; otherwise the external execution is large.

The integral part of PID operation executes conditionally and only executes when the temperature error signal $\mathrm{e}(\mathrm{k})$ is greater than $0.5^{\circ} \mathrm{C}$. Integral part is mainly used to eliminate static error, but when the temperature deviation itself is very small, adding integral coefficient can make the system response lag instead. Limit the scope of implementation of PID, in view of the heated humidification system for respirator and the features of heating plate, perform the PID arithmetic when the result of set temperature minus the actual temperature is greater than or equal to $-1^{\circ} \mathrm{C}$ and less than or equal to $5^{\circ} \mathrm{C}$; when more than $5^{\circ} \mathrm{C}$, let heating plate heating; when less than $-1{ }^{\circ} \mathrm{C}$, let heating plate does not work. This improves the efficiency of heated humidifier.

\section{Experimental results}

Based on the above PID algorithm and circuit theory, developed a prototype, and temperature stability experiment was carried out. Temperature control rendering by PID is shown on Fig. 7, set temperature are $25^{\circ} \mathrm{C}, 30^{\circ} \mathrm{C}$ and $35^{\circ} \mathrm{C}$, respectively. By the figure, we can see that temperature regulation time is short, and temperature fluctuation range is small and very stable after reach the set temperature.

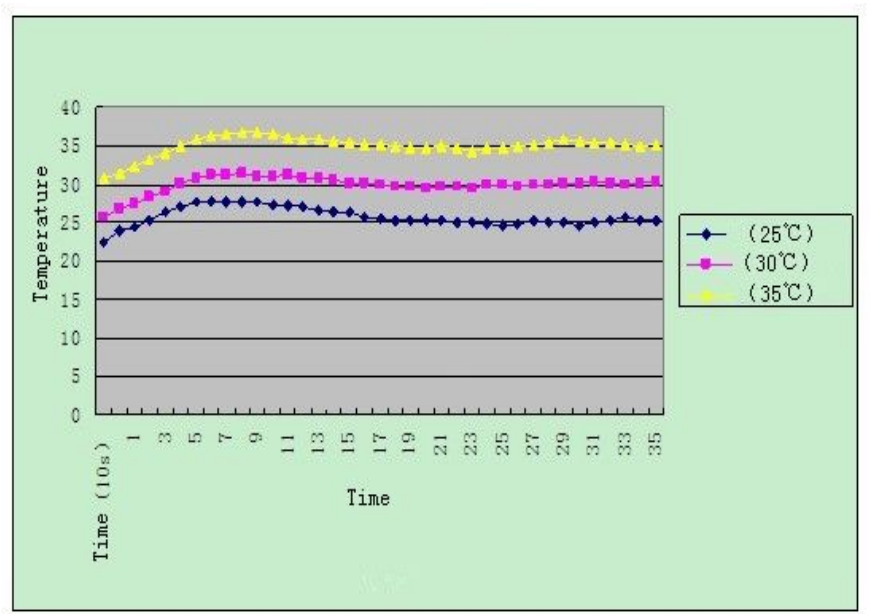

Figure7. Temperature control rendering

\section{The Conclusions}

Experimental results show that the heated humidification system in this paper meets the design requirements. It has high practical value and can be widely used in various respirator therapies. The introduction of PID algorithm makes temperature control 
high precision and responsive. Compared with the current heated humidifier, this system has the following characteristics: the temperature can be adjusted, responsive, temperature stability and security. Low cost, can be transplanted on different control chip, simple operation, and portable.

\section{References:}

[1] Fischer Y, Keck T. Effects of nasal mask leak and heated humidification on nasal mucosa in the therapy with nasal continuous positive airway pressure (nCPAP) [J]. Sleep and Breathing 2008, 12(11): 353-357.

[2] Massie CA, Hart RW, Peralez K, Richards GN (1999) Effects of humidification on nasal symptoms and compliance in sleep apnea patients using continuous positive airway pressure. Chest 116: 403-408.

[3] Martins DeAraújo MT, Vieira SB, Vasquez EC, Fleury B (2000) Heated humidification or face mask to prevent upper airway dryness during continuous positive airway pressure therapy. Chest 117: 142-147.

[4] Randerath WJ, Meier J, Genger H, Domanski U, Rühle KH (2002) Efficacy of cold passover and heated humidification under continuous positive airway pressure. EurRespir J 20:183-186.

[5] Liener K, Leiacker R, Lindemann J, Rettinger G, Keck T (2003)
Nasal mucosal temperature after exposure to cold, dry air and hot, humid air. ActaOtolaryngol 123: 851-856.

[6] Wiest GH, Fuchs FS, Brueckl WM, Nusko G, Harsch IA, Hahn EG, Ficker JH (2000) In vivo efficacy of heated and non-heated humidifiers during nasal continuous positive airway pressure (nCPAP)-therapy for obstructive sleep apnoea. Respir Med 9:364-368

[7] W. McDowell Anderson, Sherwin M. Mina, Adjunctive Therapy to CPAP: Sedative Hypnotics, Heated Humidification, and Supplemental Oxygen[J],Sleep Medicine Clinics, 2010, Vol.5 (3), pp.369-381.

[8] Astrom K J, Toward Intelligent Control. IEEE Control Systems Magazine, 1998, No.4:259-265.

[9] Radakovic Z. R, Milosevic V. M, Radakovic S. B. Application of temperature fuzzy controller in an indirect resistance furnace, Application Energy, 2002, 73:167-182.

[10] M Zhuang, D.P. Atherton. Automatic tuning of optimum PID controllers [J]. IEE Proceedings Control and Applications, 1993, No.140:216-224. 